\title{
A Size-duration Trend for Gamma-ray Burst Progenitors
}

\section{Citation}

Barnacka, Anna, and Abraham Loeb. 2014. "A SIZE-DURATION TREND FOR GAMMA-RAY BURST PROGENITORS." The Astrophysical Journal 794 (1): L8. https://doi.org/10.1088/2041-8205/794/1/ 18.

\section{Permanent link}

http://nrs.harvard.edu/urn-3:HUL.InstRepos:41412140

\section{Terms of Use}

This article was downloaded from Harvard University's DASH repository, and is made available under the terms and conditions applicable to Other Posted Material, as set forth at http:// nrs.harvard.edu/urn-3:HUL.InstRepos:dash.current.terms-of-use\#LAA

\section{Share Your Story}

The Harvard community has made this article openly available.

Please share how this access benefits you. Submit a story.

Accessibility 


\title{
A SIZE-DURATION TREND FOR GAMMA-RAY BURST PROGENITORS
}

\author{
AnNa Barnacka AND Abraham LoEb \\ Harvard-Smithsonian Center for Astrophysics, 60 Garden Street, MS-20, \\ Cambridge, MA 02138, USA; abarnacka@cfa.harvard.edu, aloeb@cfa.harvard.edu \\ Received 2014 September 4; accepted 2014 September 11; published 2014 September 25
}

\begin{abstract}
Gamma-ray bursts (GRBs) show a bimodal distribution of durations, separated at a duration of $\sim 2 \mathrm{~s}$. Observations have confirmed the association of long GRBs with the collapse of massive stars. The origin of short GRBs is still being explored. We examine constraints on the size of emission regions in short and long GRBs detected by Fermi/GBM. We find that the transverse extent of emission regions during the prompt phase, $R$, and the burst duration, $T_{90}$, are consistent with the relation $R \sim c \times T_{90}$, for both long and short GRBs. We find the characteristic transverse extent for the prompt emission region to be $\sim 2 \times 10^{10} \mathrm{~cm}$ and $\sim 4 \times 10^{11} \mathrm{~cm}$ for short and long GRBs, respectively.
\end{abstract}

Key words: gamma-ray burst: general - methods: data analysis

Online-only material: color figures

\section{INTRODUCTION}

The first catalog of gamma-ray bursts (GRBs) detected by the BATSE satellite revealed bimodality in their duration distribution (Kouveliotou et al. 1993). Events lasting longer than $2 \mathrm{~s}$ were classified as long GRBs, while those shorter than $2 \mathrm{~s}$ were classified as short GRBs. This bimodal distribution suggested a difference in physical origin and progenitor populations.

The discovery of optical afterglows following some of the long GRBs detected by BeppoSAX (van Paradijs et al. 1997; Metzger et al. 1997) and environmental studies indicated that long GRBs originate in star-forming galaxies (Bloom et al. 1998; Djorgovski et al. 1998) and their location is spatially correlated with star-forming regions within their hosts (Fruchter et al. 2006). In addition, some of the long GRBs are associated with Type Ic supernovae (Hjorth et al. 2003; Stanek et al. 2003). These clues indicate that long GRBs are associated with the core collapse of massive stars (collapsars), and not with the merger of compact object binaries (Paczyński 1998; MacFadyen \& Woosley 1999).

The origin of short GRBs is still being explored. Short GRBs are often detected at lower redshift because these bursts are less energetic. They constitute less than $20 \%$ of all detected GRBs. The lack of supernova associations shows that at least some short GRBs are not produced by massive star progenitors (Fox et al. 2005; Soderberg et al. 2006; Berger et al. 2013).

On theoretical grounds, the mergers of compact binary objects have been investigated as a possible origin of short bursts (Paczyński 1998; Eichler et al. 1989; Belczynski et al. 2006; Bogomazov et al. 2007). The compact object merger scenario is observationally supported by the location of short GRBs within their host galaxies (for a recent review, see Berger 2013).

The transition between short and long GRBs at $2 \mathrm{~s}$ is somewhat arbitrary, and it is unclear how strongly it reflects differences in the progenitors of GRBs. Bromberg et al. (2013) argued that the transition between short and long GRBs is detector dependent, and collapsars may be found among the short GRBs with some of the long GRBs produced by noncollapsars.

The radius of the emission region and its transverse extent from the central engine is crucial in understanding the origin of gamma-ray emission, and the nature of their progenitors (Zhang et al. 2003; Janiuk \& Proga 2008; Ziaeepour 2009; Beloborodov
2010; Tchekhovskoy et al. 2010). Here we investigate the constraints on the size of the emission region and beaming factor of GRBs detected by Fermi/GBM (Meegan et al. 2009). We examine the relation between the transverse extent of the prompt emission region and burst duration, its implications for short and long GRBs.

The paper is organized as follows. In Section 2, we introduce a formalism to constrain the size of the emission region. Then, in Section 3, we describe the selection of GRBs detected by Fermi/GBM. The minimum variability timescales are estimated in Section 4. The results are presented in Section 5 and discussed in Section 6. Finally, we summarize our conclusions in Section 7.

\section{SIZE OF THE EMISSION REGION}

The size of the prompt emission region is unresolved observationally. However, the light curve variability is attributed to the activity of the central engine (Rees \& Meszaros 1994; Sari $\&$ Piran 1997), and, as such, the minimum variability timescale provides a constraint on the maximum transverse extent of the emission region (relative to the line-of-sight). It is generally accepted that GRBs are powered by relativistic jets propagating with a speed $v=\beta c$ and Lorentz factor $\Gamma=\left(1-\beta^{2}\right)^{-1 / 2}$. The maximum transverse extent of the source is given by the relation

$$
\begin{aligned}
R_{\max }^{\prime} & \simeq c t_{\mathrm{var}}^{\prime} \simeq \frac{\mathcal{D} c t_{\mathrm{var}}}{(1+z)} \\
& \simeq \frac{3 \times 10^{11} \mathrm{~cm}}{(1+z)}\left(\frac{\mathcal{D}}{100}\right)\left(\frac{t_{\mathrm{var}}}{0.1 \mathrm{~s}}\right),
\end{aligned}
$$

where prime denotes quantities in the comoving frame of the emitting plasma, $t_{\mathrm{var}}$ is the observed minimum variability timescale, $z$ is the cosmological redshift, and $\mathcal{D}=[\Gamma(1-$ $\left.\left.\beta \cos \theta_{\text {obs }}\right)\right]^{-1}$ is the Doppler factor of the observed radiation, with $\theta_{\text {obs }}$ being the observer viewing angle relative to the velocity of the emitting material. For typical GRBs, $\theta_{\text {obs }} \lesssim 1 / \Gamma$ and $\mathcal{D} \sim \Gamma$.

Setting constraints on the maximum radius of the emission region requires knowledge of the Doppler factor $\mathcal{D}$. There are several methods for placing lower and upper limits on $\mathcal{D}$ (Baring et al. 1993; Baring \& Harding 1997; Lithwick \& Sari 2001; Razzaque et al. 2004; Racusin et al. 2011), which provide values in the range $20-3000$. 
The emission region has to be transparent to gamma rays. A small radius for the emission region and high photon densities imply a very large optical depth to $\gamma \gamma$ absorption and pair creation (Krolik \& Pier 1991; Baring \& Harding 1997). In order to allow gamma-ray photons to escape from the emission region, the optical depth for $\gamma \gamma$ absorption, $\tau_{\gamma \gamma}$ (Gould \& Schréder 1967), must satisfy

$$
\tau_{\gamma \gamma}(E) \simeq R^{\prime} \int_{-1}^{1} d \mu \frac{1-\mu}{2} \int_{E_{\mathrm{th}}}^{\infty} d \epsilon n(\epsilon) \sigma_{\gamma \gamma}<1,
$$

assuming isotropic emission in the comoving frame, where $R^{\prime}$ is a transverse extent of the emission region, $\mu=\cos \theta$, where $\theta$ is an angle between the momenta of the emitted photon and the ambient photon, and $\sigma_{\gamma \gamma}(E, \epsilon, \mu)$ is the polarization averaged cross-section for pair production (Jauch \& Rohrlich 1976; Boettcher 2014). Here, $E$ is the energy of the emitted photon, $\epsilon$ is the energy of the ambient photon, and $E_{\text {th }}=2 /(\epsilon(1-\mu))$ is the threshold energy for pair production. All photon energies are defined in the jet comoving frame and in dimensionless units, normalized by $m_{e} c^{2}$.

The spectral photon number density is given by

$$
n(\epsilon)=\frac{d_{L}^{2} \Phi(\epsilon)}{\mathcal{D}^{4} R^{\prime 2} m_{e} c^{3} \epsilon},
$$

where $d_{L}$ is the luminosity distance to the source, $\Phi$ is the observed energy flux (also known as $v F_{v}$ ). Combining Equations (2) and (3), assuming the transverse extent of the emission region to be $R_{\max }^{\prime}$, gives the minimum Doppler factor

$$
\mathcal{D}^{5}>\frac{(1+z) d_{L}^{2}}{c t_{\mathrm{var}}} \Upsilon
$$

where

$$
\Upsilon=\int_{-1}^{1} d \mu \frac{1-\mu}{2} \int_{E_{\mathrm{th}}}^{\infty} d \epsilon \frac{\Phi(\epsilon)}{m_{e} c^{3} \epsilon} \sigma_{\gamma \gamma} .
$$

The minimum Lorentz factor, $\Gamma_{\min }$, required for gamma rays to escape from the emission region depends on the combination of the emitted and ambient photon energies. Conservatively we evaluate $\Gamma_{\min }$ at an energy $E=2 E_{\mathrm{th}}$, where the $\gamma \gamma$ absorption cross-section has a distinct maximum. The threshold energy is calculated using the ambient photon energy, $\epsilon$, equal to the peak energy of the spectral energy distribution of the prompt emission, $E_{p}$.

Equations (2) and (3) can also be used to evaluate the minimum transverse extent of the emission region

$$
R_{\min }^{\prime} \simeq \frac{d_{L}^{2}}{\mathcal{D}^{4}} \Upsilon
$$

assuming conservatively a maximum value of $\mathcal{D} \sim 1200$ (Racusin et al. 2011).

\section{DATA SELECTION}

The Gamma-Ray Burst detector (GBM; Meegan et al. 2009) on board the Fermi satellite consists of $12 \mathrm{NaI}$ and 2 BGO scintillators which cover the energy range from $8 \mathrm{keV}$ to $40 \mathrm{MeV}$ in 128 energy bins and monitor the entire sky. In the five years of its operation, the GBM triggered on more than 1300 GRBs.

Information on the GRB distances is required for obtaining robust limits on the emission region sizes. We have therefore selected a limited sample of $\sim 50$ GRBs with measured redshifts. Of those, only bursts with good spectral quality and light curves with sufficient signal-to-noise ratio were kept. The primary condition for the data selection was at least one light curve with signal-to-noise ratios greater than 2 at a sampling of $0.128 \mathrm{~s}$. The selection procedure reduced the initial sample to 24 long GRBs with measured redshifts.

To add short GRBs, we have selected GRBs detected by the GBM with duration $T_{90}{ }^{1}$ shorter than $2 \mathrm{~s}$, and a flux greater than 15 photons $\mathrm{cm}^{-2} \mathrm{~s}^{-1}$. The flux was measured in the energy range $10-1000 \mathrm{keV}$ with $64 \mathrm{~ms}$ sampling time. Altogether, we have selected 43 short GRBs. The quality selection, the same as for long GRBs, limited the sample to 19 short GRBs. The entire sample of short GRBs has unknown redshifts, and so we have assumed an average redshift of 0.85 (D'Avanzo et al. 2014).

The light curve analysis and a spectra fitting in our analysis have been performed following the procedures described by Holland et al. (2012). ${ }^{2}$

\section{MINIMUM VARIABILITY TIMESCALE}

The Fermi/GBM triggers on bursts within $16 \mathrm{~ms}$. All triggers generate time-tagged event data consisting of the photon arrival time and energy as deposited in each of the 14 detectors with a temporal resolution of $2 \mu$ s (Meegan et al. 2009).

The intrinsic minimum variability timescale of GRBs is determined by the size of the emission region and the emission mechanism. The observed minimum variability time scales are in addition limited by the sensitivity of the detectors, the GRBs flux, and the sampling of light curves. Figure 1 shows the light curve of one of the brightest GRBs detected by GBM: GRB090424. The light curve is displayed with two different binnings, $0.128 \mathrm{~s}$ and $0.01 \mathrm{~s}$, demonstrating how binning can smooth variability or decrease signal-to-noise ratio.

To find the observed minimum variability time scale for each GRB, we have used the method utilized by Bhat et al. (2012) and Bhat (2013a, 2013b), which searches for a characteristic timescale at which the variance ratios per bin width is minimum. The characteristic timescale is interpreted as an upper limit on the minimum variability timescale. This method incorporates the following steps: first, the time interval of the prompt emission is selected based on $T_{90}$; then, a background time interval of an equal duration is selected. Both the signal and the background intervals are used to derive differentials which, in the next step, are used to calculate variances of the signal and the background. The ratios of the variances are calculated for different binnings in the range from $10^{-3} \mathrm{~s}$ up to $0.1 \times T_{90}$ using ten logarithmic bins per decade. The bin width at which the variance ratio divided by the bin width obtains its minimum value is interpreted as a minimum observed variability timescale, $t_{\text {var }}$ (e.g., see Figure 1 in Bhat 2013a). The resulting minimum variability timescales for the entire sample are listed in Table 1.

The variance ratio contains information on the rates of change in the light curves. These rates of change can decrease either because of the lack of further variability of the sources, or due to limited photon statistics.

To assess whether the observed minimum variability time scale can be intrinsic to the source, we have calculated an

\footnotetext{
$1 T_{90}$ is defined as the time interval over which $90 \%$ of the flux, integrated over the burst duration, was detected (http://heasarc.gsfc.nasa.gov/W3Browse/ fermi/fermigbrst.html\#t90).

2 http://fermi.gsfc.nasa.gov/ssc/data/analysis/user/do_gbm.pdf
} 


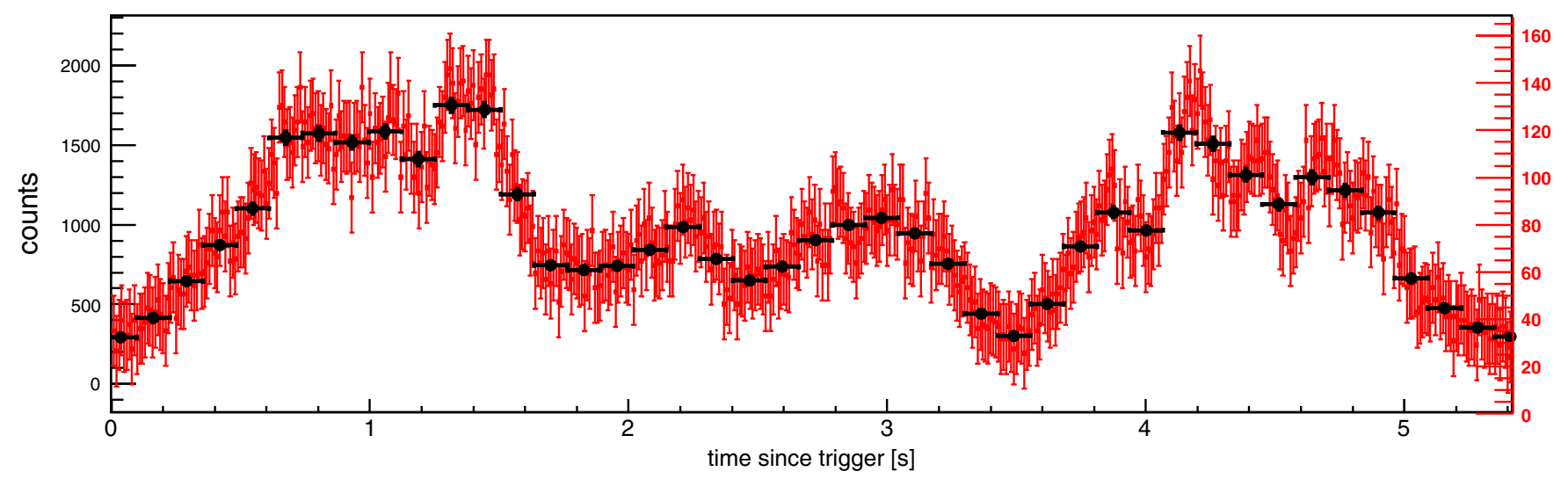

Figure 1. Sample light curve of GRB090424 using two binnings; 0.128 s represented by black points, and 0.01 by red points.

(A color version of this figure is available in the online journal.)

average significance of the signal in the light curve for each binning, $\hat{N}_{\sigma}$. If the average significance at the observed minimum variability time scale is $>4 \sigma$, the observed variability time scale is interpreted as the intrinsic property of the emission region.

The average significance, $\bar{N}_{\sigma}$, of the light curve with a binning corresponding to $t_{\mathrm{var}}$ is listed in Table 1 for samples of long and short GRBs.

We have found that the average minimum variability timescale obtained for short GRBs is $0.036 \mathrm{~s}$ and $1.2 \mathrm{~s}$ for long GRBs. In our samples, the observed variability time scales for long GRBs are significantly longer than those of short GRBs. This is in agreement with the minimum variability timescales of long and short GRBs obtained by MacLachlan et al. (2013).

The high significance obtained at $t_{\mathrm{var}}$ suggests that for the majority of the long GRBs in our sample, the observed variability timescale is not limited by the statistics. In the case of short GRBs, the average significance at the observed minimum variability timescale is small, suggesting that the intrinsic variability timescales for these GRBs can be shorter than observed.

MacLachlan et al. (2013) noticed a correlation between the minimum variability timescale and the burst duration for short GRBs. A hint of such a correlation is also present in our sample. However, as we have shown, the average significance at the characteristic variability timescale is small for short GRBs. Thus, the correlation can be the result of limited photon number statistics.

In our sample, the observed minimum variability timescales for long GRBs does not show a correlation with the duration of the burst, $T_{90}$. This is in agreement with Golkhou \& Butler (2014), who investigated the minimum variability timescales for a large sample of Swift GRBs.

\section{RESULTS}

Following the formalism described in Section 2, we have obtained the maximum and minimum transverse extents of the prompt emission regions for a sample of 19 short and 24 long GRBs. Table 1 lists the properties for each burst.

Figure 2 shows upper and lower limits on the transverse extent of the emission region as a function of the duration of the engine activity $T_{90} /(1+z)$. The upper limits were obtained using Equation (1), where the minimum variability time scale was derived from the light curve analysis described in Section 4, and the Doppler factor was constrained using Equation (4). The

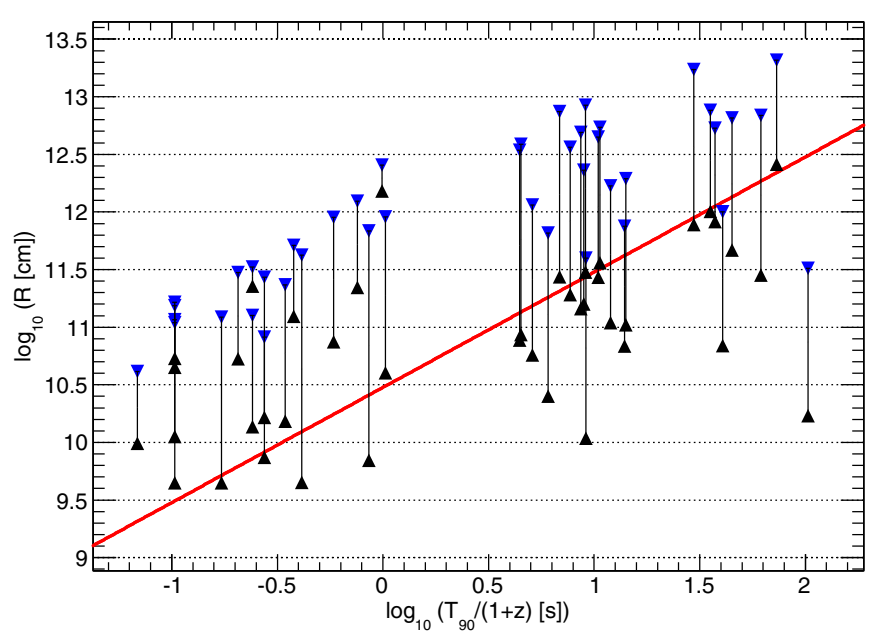

Figure 2. Upper and lower limits on the transverse extent of the emission region as a function of $T_{90} /(1+z)$. Blue down-pointing triangles indicates upper limits and black up-pointing triangles corresponds to lower limits. The red line equates the transverse extent of the emission region to $c \times T_{90} /(1+z)$, where $T_{90} /(1+z)$ is an engine lifetime. Note that this line is not attempting to fit the constraints from the data.

(A color version of this figure is available in the online journal.)

energy flux, $\Phi(\epsilon)$, was obtained by fitting the Band function (Band et al. 1993) to the spectrum.

\section{DISCUSSION}

Our constraints on the transverse extent of prompt emission region is based on the analysis of the minimum variability time scales and the Doppler factors, which are evaluated using $T_{90}$ and the same energy range for all GRBs.

The luminosity distances in the sample of long GRBs have been calculated using measured redshifts, whereas for the sample of short GRBs we have assumed an average redshift of 0.85 (D'Avanzo et al. 2014). The constraints on the Doppler factor scale with the luminosity distance as $\mathcal{D} \alpha d_{L}^{2 / 5}$. Even if short GRBs are located five times farther away, the observed size would be underestimated by merely a factor of two. The maximum transverse extent of the emission regions of long GRBs is larger than that of short GRBs by over an order of magnitude. Thus, the assumed redshift for short GRBs has little impact on our results.

The minimum transverse extent of the emission region has been estimated differently for short and long GRBs. For the 
Table 1

Sample of Short and Long GRBs

\begin{tabular}{|c|c|c|c|c|c|c|c|c|}
\hline Name & $\begin{array}{c}\text { Redshift } \\
\text { (z) }\end{array}$ & $\begin{array}{l}T_{90} \\
(\mathrm{~s})\end{array}$ & $\begin{array}{l}T_{50} \\
(\mathrm{~s})\end{array}$ & $\begin{array}{r}t_{\mathrm{var}} \\
(\mathrm{s})\end{array}$ & $\bar{N}_{\sigma}$ & $\mathcal{D}_{\text {min }}$ & $\begin{array}{l}R_{\min } \\
(\mathrm{cm})\end{array}$ & $\begin{array}{l}R_{\max } \\
(\mathrm{cm})\end{array}$ \\
\hline RB081209981 & & & & 0. & 3 & 30 & & \\
\hline RB081216531 & & & 0.128 & & 0.4 & 390 & & \\
\hline RB090108020 & $\cdots$ & 0.704 & 0.256 & 0.038 & 0.2 & 850 & & \\
\hline RB090228204 & $\cdots$ & 18 & 0.1 & 0.0 & 0.4 & 700 & & \\
\hline RB090328713 & $\cdots$ & 0.18 & 0.128 & 0.011 & 0.2 & 680 & & \\
\hline 35 & $\ldots$ & & 0.064 & & 0.4 & 850 & & \\
\hline RB100929916 & $\ldots$ & 0.320 & 0.256 & 0.014 & 0.1 & 530 & & $1.2 \mathrm{e}+11$ \\
\hline RB & $\ldots$ & 1.917 & 0.512 & 0.1 & 0.4 & 560 & & \\
\hline RB 1107 & $\cdots$ & 0.17 & 0.128 & 0. & 0.6 & 910 & & \\
\hline $\mathrm{SRB}$ & $\cdots$ & & & & 0.2 & 890 & & \\
\hline RB & & & & & 1.1 & 650 & & \\
\hline 0323507 & $\ldots$ & 0.4 & 0.192 & 0.0 & 3.3 & 1100 & & 3.3 \\
\hline$R B$ & $\ldots$ & 0.640 & 0.1 & & 0.8 & 610 & & \\
\hline $\mathrm{RE}$ & $\cdots$ & 0.19 & 0.0 & & 0.2 & 550 & & \\
\hline $\mathrm{RF}$ & & & & & 0.6 & 780 & & \\
\hline SRB130628860 & $\cdots$ & 0.512 & 0.384 & 0.008 & 0.2 & 670 & +09 & $8.2 \mathrm{e}+10$ \\
\hline GRB1 & $\ldots$ & 1.600 & 0.7 & 0.1 & 3.5 & 390 & & \\
\hline $\mathrm{SRB}$ & & & & & 2.0 & 600 & & \\
\hline $\mathrm{SRE}$ & . & 8 & 0 & 0. & 1.3 & 780 & & \\
\hline $\mathrm{RBO}$ & 2205 & .704 & & 21 & 6.3 & 380 & $e+10$ & \\
\hline$R B$ & 0.689 & 7 & .000 & 23 & 10.2 & 30 & 10 & \\
\hline $\mathrm{sRB}$ & 4 & & 2 & 1. & 2.1 & 80 & 10 & \\
\hline 8 & & & & & 0.5 & & & \\
\hline 1681 & 2.260 & .697 & 7. & 1. & 8.6 & 560 & & +12 \\
\hline 204 & 2.700 & 30 & 4.6 & 0.3 & 11.3 & 400 & & 1. \\
\hline GRH & & & & & 3 & & & \\
\hline 002 & & 135 & 53. & 5.943 & 11.0 & 440 & & +13 \\
\hline 01 & 0.736 & 697 & 14.592 & 0.3 & 4.5 & 150 & -10 & \\
\hline GRB & 0.544 & 4.144 & 2 & 0. & 7.0 & 30 & 08 & 3. \\
\hline GRB0 & & & & & 3.0 & 60 & & \\
\hline GRB090902462 & 1.822 & & 9.0 & & 7.8 & 540 & & \\
\hline GRB & 2.106 & 13.760 & 6.5 & 0.3 & 8.8 & 890 & 10 & $3.4 \mathrm{e}+12$ \\
\hline GRBC & & & 13.3 & & 3.1 & 0 & & $5.4 \mathrm{e}+12$ \\
\hline GRB091020900 & & & & & 0.5 & 290 & $e+10$ & $2.3 e+12$ \\
\hline 72 & 1.0 & & 39.9 & & 1.5 & 280 & $4.9 \mathrm{e}+10$ & $6.5 e+12$ \\
\hline GRB091208410 & 1.063 & 12.480 & 7.168 & 0.087 & 0.5 & 520 & $2.6 e+09$ & $6.5 \mathrm{e}+11$ \\
\hline GRB100814160 & 1.440 & 150.53 & 72.193 & 1.148 & 12.0 & 490 & $3.4 e+10$ & $6.9 \mathrm{e}+12$ \\
\hline GRB100906576 & 1.727 & 110.59 & 18.944 & 0.3 & 7.6 & 290 & $e+09$ & $1.0 \mathrm{e}+12$ \\
\hline GRB110213220 & 1.460 & 34.305 & 6.400 & 0.281 & 3.5 & 220 & $8.4 \mathrm{e}+09$ & $7.5 \mathrm{e}+11$ \\
\hline GRB120712571 & 4.000 & 22.528 & 7.424 & & 0.5 & 900 & & $3.9 e+12$ \\
\hline GRB120729456 & 0.800 & 25.472 & 8.320 & 0.316 & 3.0 & 370 & $9.5 e+09$ & $1.9 \mathrm{e}+12$ \\
\hline GRB130427324 & & 138.24 & 4.096 & 0.038 & 7.5 & 380 & $1.1 \mathrm{e}+09$ & $3.2 \mathrm{e}+11$ \\
\hline GRB130518551 & 2.490 & 3.456 & 1.280 & 0.280 & 3.1 & 1060 & $1.5 e+12$ & $2.5 \mathrm{e}+12$ \\
\hline
\end{tabular}

Note. Column labels denote the names of the GRBs, GRB duration $\left(T_{90}\right)$, minimum variability timescale $\left(t_{\mathrm{var}}\right)$, the average significance of the signal in the light curve with binning corresponding to $t_{\mathrm{var}}$, the minimum Doppler factor $\left(\mathcal{D}_{\min }\right)$, the minimum size of the emission region $\left(R_{\min }\right)$, and the maximum size of the emission region $\left(R_{\max }\right)$.

majority of the long GRBs, the observed minimum variability timescale is not limited by the photon counting statistics, and thus may refer to the intrinsic variability of the source. Therefore, the minimum transverse extent of the emission regions has been calculated as $\Gamma_{\min } c t_{\mathrm{var}} /(1+z)$, assuming conservatively a minimum value of Lorenz factor, $\Gamma_{\min }, \sim 20$ (Ghirlanda et al. 2012; Sonbas et al. 2014).

The observed variability timescale for short GRBs is limited by photon statistics; thus, the emission region can be smaller than estimated. To evaluate the minimum transverse extent of the emission region in the sample of short GRBs, we have used Equation (6).

The emission region of GRBs is expected to expand close to the speed of light. We have therefore compared our results to

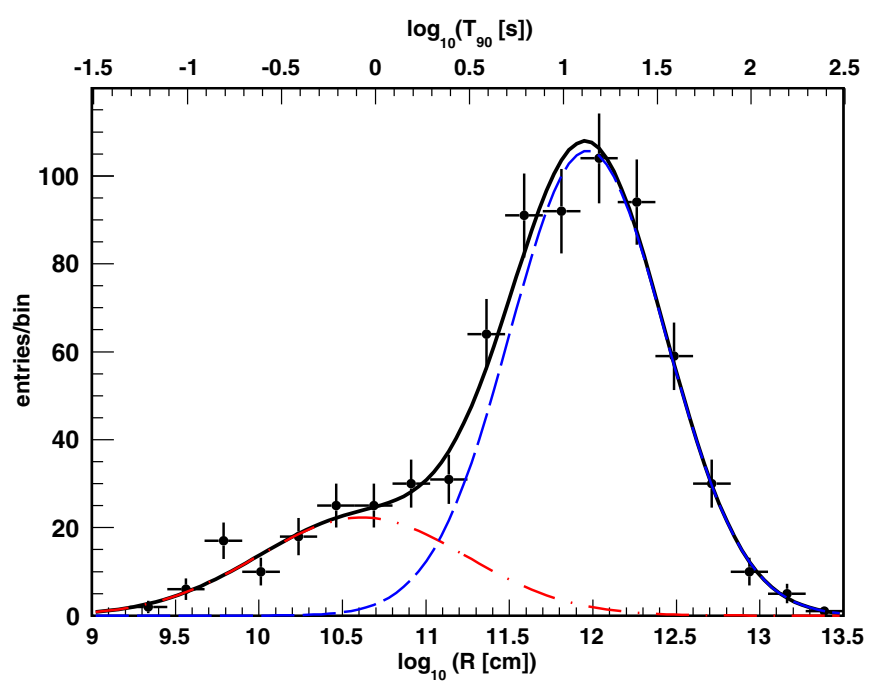

Figure 3. Size distribution of emission regions during the prompt emission of GRBs. The distribution is obtained for a total of 1349 GRBs detected by Fermi/GBM. The transverse extents are estimated using the duration $T_{90}$ multiplied by the speed of light, without correcting for the $(1+z)$ redshift factor. The solid line delineates a fit to the distribution involving two Gaussian functions (in blue and red).

(A color version of this figure is available in the online journal.)

$c \times T_{90}^{\prime}$. Remarkably, this relation lies within the constrained transverse extent of the emission region of the sample of long and short GRBs.

Figure 3 shows the transverse extents distribution of the emission regions of all GRBs detected by the GBM detector until 2014 June. The transverse extents have been estimated using the relation $c \times T_{90}$. The distribution of transverse extents is bimodal with an average transverse extent of the emission regions of $\sim 2 \times 10^{10} \mathrm{~cm}$ and $\sim 4 \times 10^{11} \mathrm{~cm}$ for short and long GRBs, respectively. These transverse extents are corrected for the redshift factor assuming an average redshift of $z=0.85$ (D'Avanzo et al. 2014) for short GRBs and an average redshift of $z=1.77$ for long GRBs listed in Table 1 .

\section{SUMMARY}

The bimodality in the distribution of GRB durations has been interpreted as evidence for two progenitor populations. In the collapsar scenario, the central engine has to be active long enough for the emission region to exit the stellar envelope and produce the observed $\gamma$-rays. In this scenario, the radius of the stellar envelope is $\lesssim 10^{12} \mathrm{~cm}$.

Our constraints imply that the emission region size during the prompt GRB emission, $R$, and the central engine duration, $T_{90}$, are consistent with the relation $R \sim c \times T_{90}$. We have obtained the characteristic transverse extent of the prompt emission region to be $\sim 2 \times 10^{10} \mathrm{~cm}$ and $\sim 4 \times 10^{11} \mathrm{~cm}$ for short and long GRBs, respectively.

The average beaming factor for short GRBs in our sample is 670 , yielding a radius of emission region, $r \sim \Gamma R$, of $\sim 10^{13} \mathrm{~cm}$. The average beaming factor for long GRBs is 450 , implying the radius of emission region of $\sim 2 \times 10^{14} \mathrm{~cm}$.

We thank the referee for valuable comments on the manuscript. We thank Markus Böttcher, Edo Berger, Josh Grindlay, and Raffaella Margutti for comments on the manuscript and for useful discussions. The work of A.B. is supported by the Department of Energy Office of Science, NASA, and the Smithsonian Astrophysical Observatory, and financial 
support by the NCN grant DEC-2011/01/M/ST9/01891 is acknowledged. This work was also supported in part by NSF grant AST-1312034.

\section{REFERENCES}

Band, D., Matteson, J., Ford, L., et al. 1993, ApJ, 413, 281

Baring, M. G., Ellison, D. C., \& Jones, F. C. 1993, ApJ, 409, 327

Baring, M. G., \& Harding, A. K. 1997, ApJ, 491, 663

Belczynski, K., Perna, R., Bulik, T., et al. 2006, ApJ, 648, 1110

Beloborodov, A. M. 2010, MNRAS, 407, 1033

Berger, E. 2013, ARA\&A, 52, 43

Berger, E., Fong, W., \& Chornock, R. 2013, ApJL, 774, L23

Bhat, N. P. 2013a, in EAS Publications Series, Vol. 61, The Seventh Huntsville Gamma-Ray Burst Symposium, ed. A. J. Castro-Tirado, J. Gorosabel, \& I. H Park (Paris: ESA), 45

Bhat, P. N. 2013b, arXiv:1307.7618

Bhat, P. N., Briggs, M. S., Connaughton, V., et al. 2012, ApJ, 744, 141

Bloom, J. S., Djorgovski, S. G., Kulkarni, S. R., \& Frail, D. A. 1998, ApJL, 507, L25

Boettcher, M. 2014, ApJ, in press (arXiv:1409.1674)

Bogomazov, A. I., Lipunov, V. M., \& Tutukov, A. V. 2007, ARep, 51, 308

Bromberg, O., Nakar, E., Piran, T., \& Sari, R. 2013, ApJ, 764, 179

D’Avanzo, P., Salvaterra, R., Bernardini, M. G., et al. 2014, arXiv:1405.5131

Djorgovski, S. G., Kulkarni, S. R., Bloom, J. S., et al. 1998, ApJL, 508, L17

Eichler, D., Livio, M., Piran, T., \& Schramm, D. N. 1989, Natur, 340, 126

Fox, D. B., Frail, D. A., Price, P. A., et al. 2005, Natur, 437, 845
Fruchter, A. S., Levan, A. J., Strolger, L., et al. 2006, Natur, 441, 463

Ghirlanda, G., Nava, L., Ghisellini, G., et al. 2012, MNRAS, 420, 483

Golkhou, V. Z., \& Butler, N. R. 2014, ApJ, 787, 90

Gould, R. J., \& Schréder, G. P. 1967, PhRv, 155, 1408

Hjorth, J., Sollerman, J., Møller, P., et al. 2003, Natur, 423, 847

Janiuk, A., \& Proga, D. 2008, ApJ, 675, 519

Jauch, J. M., \& Rohrlich, F. 1976, The Theory of Photons and Electrons. The Relativistic Quantum Field Theory of Charged Particles with Spin One-half (New York: Springer)

Kouveliotou, C., Meegan, C. A., Fishman, G. J., et al. 1993, ApJL, 413, L101

Krolik, J. H., \& Pier, E. A. 1991, ApJ, 373, 277

Lithwick, Y., \& Sari, R. 2001, ApJ, 555, 540

MacFadyen, A. I., \& Woosley, S. E. 1999, ApJ, 524, 262

MacLachlan, G. A., Shenoy, A., Sonbas, E., et al. 2013, MNRAS, 432, 857

Meegan, C., Lichti, G., Bhat, P. N., et al. 2009, ApJ, 702, 791

Metzger, M. R., Djorgovski, S. G., Kulkarni, S. R., et al. 1997, Natur, 387, 878

Paczyński, B. 1998, ApJL, 494, L45

Racusin, J. L., Oates, S. R., Schady, P., et al. 2011, ApJ, 738, 138

Razzaque, S., Mészáros, P., \& Zhang, B. 2004, ApJ, 613, 1072

Rees, M. J., \& Meszaros, P. 1994, ApJL, 430, L93

Sari, R., \& Piran, T. 1997, ApJ, 485, 270

Soderberg, A. M., Berger, E., Kasliwal, M., et al. 2006, ApJ, 650, 261

Sonbas, E., Dhuga, K. S., Veres, P., et al. 2014, arXiv:1408.3042

Stanek, K. Z., Matheson, T., Garnavich, P. M., et al. 2003, ApJL, 591, L17

Tchekhovskoy, A., Narayan, R., \& McKinney, J. C. 2010, NewA, 15, 749

van Paradijs, J., Groot, P. J., Galama, T., et al. 1997, Natur, 386, 686

Zhang, W., Woosley, S. E., \& MacFadyen, A. I. 2003, ApJ, 586, 356

Ziaeepour, H. 2009, MNRAS, 397, 386 